\title{
The growing threat of antibiotic resistance in the Eastern Mediterranean Region - what does it take to control it?
}

\author{
R. Hajjeh ${ }^{7}$ and A. Mafi ${ }^{1}$
}

For decades, antibiotics represented an essential component of modern medicine. Not only were they responsible for saving millions of lives from previously deadly infections, but also made it possible to perform complex surgeries and better manage complications of chemotherapy and diabetes, among others. However, these miracle drugs are quickly losing their power and becoming ineffective, as bacteria have increasingly become resistant to them. The global rates of antimicrobial resistance (AMR) have become alarmingly high with dire consequences: over 700000 deaths worldwide attributable to antibiotic resistant bacteria (1). In the Eastern Mediterranean Region (EMR), reported rates of resistance have reached dangerous levels both in healthcare settings and the community, threatening the hard-won gains in health and development, and the sustainability of public health response to many communicable diseases, including pneumonia, diarrhoea, tuberculosis, malaria, HIV/AIDS, sexually transmitted infections and healthcare acquired infections.

A systematic review on the burden of AMR in the EMR conducted in 2015 (WHO /EMRO, unpublished data) revealed significant gaps in studies representative of national burden of antimicrobial disease, particularly from lower and middle-income countries (LMICs), where the impact of AMR is more detrimental. In particular, the viability of achievements through the health-related Millennium
Development Goals, and subsequently, achieving the Sustainable Development Goals, are at great risk. Multiple factors are responsible for the rise of antibiotic resistance, with the main driving factor being the widespread overuse and misuse of antibiotics in both the health and agriculture sectors.

\section{Global response to antibiotic resistance}

In order to address the growing threat of antibiotic resistance, a global coordinated effort involving all countries and key stakeholders has been initiated. In May 2015, a Global Action Plan on AMR (2) was adopted by countries at the World Health Assembly and supported by the governing bodies of the Food and Agriculture Organization (FAO) and the World Organisation for Animal Health (OIE). Furthermore, recognizing the catastrophic consequences of failing to tackle AMR, world leaders gathered at the United Nations General Assembly (UNGA) in New York in September 2016 and committed to taking a broad coordinated approach to address the root causes of AMR across multiple sectors (3). Countries are expected to report on their progress to the UNGA in 2018.

\section{Taking Action in the EMR}

\section{Raising awareness}

One of the key objectives of the global action plan is to improve awareness and understanding of AMR through effective communication, education and training. Increasing awareness is critical in order to understand the root causes of AMR and the measures needed to control it, including more cautious use of antibiotics, better hygiene and infection control, and better prevention of infection through the use of vaccines.

In 2015, WHO launched a global campaign to increase awareness of antibiotic resistance and to encourage best practices among the general public, health workers and policy makers to avoid the further emergence and spread of antibiotic resistance. The theme of the campaign, "Antibiotics: Handle with Care", reflects the overarching message that antibiotics are a precious resource and should be preserved. They should be used to treat bacterial infections, only when prescribed by a certified health professional. This campaign continues this year and the week of 14-18 November, 2016 is the second global Antibiotics Awareness Week. $\mathrm{WHO}$ is encouraging all countries, health partners, and the public to join this campaign and help raise awareness of antibiotic resistance.

\section{Developing national AMR plans}

Resolution WHA 68.7 of the World Health Assembly urged all countries to develop and have in place by 2017 national action plans on AMR that are aligned with the objectives of the global action plan. These plans are important as they serve as road maps for countries to guide their efforts to curb antibiotic resistance. So far, 12 countries in the Region have started developing their 
national action plans, with technical assistance provided by WHO, as needed.

\section{Surveillance of resistance}

Although data on AMR are increasingly being generated from the region (4-6), there are still significant gaps in the information available on antibiotic resistance. To understand better and respond to antibiotic resistance patterns and key drivers, standardized information about AMR incidence, prevalence, and trends must be gathered.

Surveillance is the cornerstone for assessing the burden of AMR and for providing the necessary information for action in support of local, national and global strategies. In order to inform decision-making and provide the evidence base for action and advocacy, WHO launched the Global
Antimicrobial Resistance Surveillance System (GLASS), which supports a standardized approach to the collection, analysis and sharing of data on AMR at a global level (7). Countries in the EMR are increasingly recognizing the importance of GLASS, and have initiated the process needed to establish this surveillance system. GLASS will help generate more data from the region that are comparable across countries, and will make it possible to monitor the impact of various interventions that aim at reducing rates of AMR.

\section{Call to action}

The adoption of the Global Action Plan on AMR through resolution WHA 68.7 and the UNGA political declarations on AMR are important milestones, but this is just the beginning. Real change depends on the governments and other partners to enact effective public policies, legislations, multisectoral collaboration, and to encourage new drugs and diagnostics development. Antibiotic resistance affects all of us-from healthcare facilities to communities, families and individuals the world over. It demands immediate and aggressive action by everyone. Without political commitment at all levels: community, healthcare workers, animal health, industry and others, we are headed for a post-antibiotic era where minor infections once again kill, and the benefits of modern medicine infectious diseases will disappear. Our region in particular has suffered from many wars and conflicts recently that have led to a reemergence of many infectious diseases (8). We cannot afford losing war against antibiotic resistance.

\section{References}

1. Jim O’Neill. Tackling Drug-Resistant Infections Globally: Final Report and Recommendations. The Review on Antimicrobial Resistance. London: the UK Government and the Wellcome Trust, May 2016 (ttps://amr-review.org/Publications).

2. Global Action Plan on AMR. Geneva: World Health Organization, 2015 (ISBN: 9789241509763) (http://www.who.int/antimicrobial-resistance/ publications/global-action-plan/en/).

3. PRESS RELEASE. High-Level Meeting on Antimicrobial Resistance. New York: UN Headquarters, 2016 (http://www. un.org/pga/71/2016/09/21/press-release-hl-meeting-onantimicrobial-resistance/, accessed 8 November 2016).

4. El-Herte RI, Kanj SS, Matar GM, Araj GF. The threat of carbapenem-resistant Enterobacteriaceae in Lebanon: an update on the regional and local epidemiology. J Infect Public Health. 2012;5:233-43.
5. Saied T, Elkholy A, Hafez SF, Basim H, Wasfy MO, El-Soubary $\mathrm{W}$, et al. Antimicrobial resistance in pathogens causing nosocomial bloodstream infections in university hospitals in Egypt. Am J Infect Control. 2011;39:e61-5.

6. Aly $\mathrm{M}$, Balkhy $\mathrm{HH}$. The prevalence of antimicrobial resistance in clinical isolates from Gulf Corporation Council countries. Antimicrob Resist Infect Control. 2012;1:26.

7. Global Antimicrobial Resistance Surveillance System, Manual for Early Implementation. Geneva: World Health Organization, 2015 (ISBN 978924154940 0) (http://www.who.int/ antimicrobial-resistance/global-action-plan/surveillance/ glass/en/).

8. Alwan A. The cost of war. Newsweek Nov. 5, 2015. http:// newsweekme.com/the-cost-of-war). 